\title{
Digital Facilitation Assistance for Collaborative, Creative Design Processes
}

\author{
Eva A. C. Bittner \\ University of Hamburg \\ bittner@informatik.uni-hamburg.de
}

\author{
Milad Mirbabaie \\ University of Bremen \\ milad.mirbabaie@uni-bremen.de
}

\author{
Stefan Morana \\ Saarland University \\ stefan.morana@uni-saarland.de
}

\begin{abstract}
People focus more and more on creating innovations collaboratively. Digital assistants (DAs) can accelerate such collaborative, creative design processes by supporting people in their work. Especially in the context of design, such as design thinking, moderators that facilitate collaborative, creative workshops can benefit from the support for their teams and themselves in the form of a DA. Based on interviews with experienced workshop facilitators from research and practice, we discuss implications for the design and usage of DAs in collaborative, creative design processes. We identify 16 distinct capabilities of DAs for task, process and interaction facilitation to guide design research and practitioners' endeavors toward helpful automated DT facilitation support. Moreover, we outline a research agenda to foster future research on this young research area.
\end{abstract}

\section{Introduction}

Over the last years, collaboration has taken new facets and people are focusing more and more on creating innovations collaboratively. To orchestrate this collaboration, innovation approaches, such as Design Thinking (DT), have been established to involve stakeholders with different backgrounds [18, 42]. Nowadays DT describes a problem-solving approach that can be divided into different stages. The amount of stages can vary with different authors from five to seven [3, 69]. DT is a framework, which integrates various tools and techniques for problem solving [42]. Because of its flexibility, multitude of methods, and a necessary open mindset, DT processes usually rely on facilitation by a skilled DT coach and are challenging for facilitators and participants alike. Thus, DT attracted interest from academics and practitioners [46]. Different studies on DT approaches tested the potential usage of information technology (IT) to reduce the effort of the people invol- ved, improve the overall experience, and resulting outcomes. For example, Rauth et al. [50] found that the creation of dedicated DT spaces was important for revealing values of experimentation. The creation of these spaces in a virtual environment was further researched and allowed the participants to engage in the process without being at the same location [35].

Another approach to enhance the DT process is to include digital assistance systems. In their article, Zhu et al. [15] revealed that the research phase could be accelerated by including artificial intelligence (AI), e.g. in the form of a digital assistant (DA), which led to shortening of the time dedicated for this activity [21]. While AI in the form of DAs can take over different roles in collaboration (e.g. peers), in the study at hand we focus on DAs that act as facilitators [10]. Specific DAs have been tested before as a facilitator in a virtual group brainstorming session to support and organize it $[8,62]$ or as an automated facilitator for the Empathy Map Method in DT [9]. The used DA performed successfully in moderating the session. However, with advances in natural language processing and machine learning, more potentials of machines taking over facilitation tasks arise, allowing human participants to focus more on their value-creating creative work. The informed design of facilitation support for DT also demands design knowledge considering the complexity of the entire DT process and grounded in real-world needs of facilitators and participants of collaborative creative design processes [57]. In our research project, we contribute to this research stream and address the following research question:

How can collaborative, creative design proces-

ses such as Design Thinking be supported with

DA systems serving as automated facilitators?

The paper proceeds with conceptual foundations and related work. Next, we outline the research approach and present results from our interview study. We discuss implications and propose avenues for further research on the support of collaborative, creative design processes with DAs. 


\section{Foundations and Related Work}

\subsection{Collaborative, creative design processes and Design Thinking}

Research revealed, that the phenomenon of communication is the driving force for collaborative creativity [59]. For example, the process of sharing or criticizing ideas, will likely encourage creativity among individuals in teams and hence lead to the creation of new ideas, which is the determination of collaborative creativity. Therefore, creative collaborators can be seen as: "people who are interacting with others to discover genuinely new ways of thinking and doing something new together. Collaborators play a principal role by conceiving and carrying out the work [...]" [60].

Collaborative creative design processes appeared due to the increasing complexity and growing number of design projects [71, 72]. They support employees to work together more efficiently for a satisfactory result [12]. One of several approaches for supporting such processes, which is used to help solving complex problems is called Design Thinking (DT) [22]. It aims at inventing new patterns, instead of analyzing them, and realizing new possibilities [28]. DT is described as a cross-disciplinary and user centered method, which proposes to work in teams with an interdisciplinary background [43]. If executed well, the DT can lead to increased team collaboration [26]. DT also aims to let designers participate more in the whole process, enable them to see the big picture and look upon the economic bottom line [11]. The process of DT is often articulated to the Stanford University's d.school 5-step approach [63], an adaption of a design process activity [5]. It consists of five recursive steps: empathize, define, ideate, prototype, and test [28, 63]. In previous work it has been observed that it can be challenging for a project manager to understand the users' real problem correctly [27]. By conducting interviews with users, for example, the first step "empathize" can avoid project failure, as its intention is to minimize the risk of misunderstandings [55]. The aim of the second stage "define", is to transfer the knowledge gained previously into one or multiple problem statements. The "ideate" stage is characterized by the creation of a large number of ideas without any judgement made by others [34]. However, during this step the previously identified problem(s) should not be lost from sight. Based on this, prototypes are created in stage four. In the last "test" stage the representations of the previously developed prototypes are tested by users. In contrast to other approaches, the design is tested early [11] in order to deduce what users see as central functions of the product. In addition, testing aims to reveal, which elements are classified as rather unimportant or even unnecessary [56]. If the test shows different results than expected, the stages can be repeated with new assumptions. In order to obtain the best result possible, the process is intentionally iterative. Through the multiple passes of the five stages, several solutions are tested until the optimal one is found [20, 28, 31]. DT projects are high-value, but complex collaborative endeavors, which to date mostly rely on expert human coaches. However, little is known about how to use DAs within collaborative, creative processes.

\subsection{DAs in Creative Design Processes}

Basic DAs are language-enabled software, which performs basic, information-based tasks for its users [19]. These tasks can serve several purposes, such as entertainment, home automation, and task management like timers and reminders. The majority of DAs rely on a conversational user interface instead of or in addition to the classic graphical user interface [44]. Therefore, the term DA is often used synonymously with the terms chatbot, conversational agent, or dialogue system, which all refer to the usage of natural language for the interaction between users and the system. Thereby, the interaction is based on using written and/or spoken language, resulting in text-based (i.e. chatbots) and speech-based systems (e.g. Siri or Alexa) [2, 25, 32, 41]. However, it has to be noted that DAs can include nonconversational capabilities as well, such as expert systems, intelligent information dashboards, or intelligent search. An important enabler of DAs is the usage of AI. By applying AI technologies, like machine learning, DAs "augment human task performance with higher extents of interactivity and intelligence than previous generations of DAs or traditional software applications" [44]. Although the utilization of DAs at the workspace is still limited in contrast to the use in private life, their importance in work related settings is evolving [64]. Over the last years the usage of DAs to improve collaboration at the workspace has been gradually getting more attention $[30,37,58]$. DAs are utilized in different branches and for various tasks, e.g. for internal personnel communication [54], education purposes [33] and customer services on social media $[15,70]$. Moreover, companies use DAs in order to get reminders for upcoming meetings or deadlines [61]. Nevertheless, a recent study revealed that even at the large business sector only $24 \%$ of companies currently use DAs, for tasks such as voice dictation or calendar management [65]. However, Gartner [47] predicts that by 2021 , one quarter of all digital workers will use a DA daily.

When it comes to creative collaboration, the question arises how DAs can be utilized for supporting and facilitating the collaboration process. Recent empirical and design work started to address specific aspects of 
this question [10]. Bittner and Shoury [9] could present results, in which a chatbot performed successfully in facilitating a method within DT. This is also confirmed by previous studies, which present similar conclusions by showing DAs' potential [2, 16, 68]. Wang et al. [55] have also shown that brainstorming is more effective within a DA-human interaction than in a human-human interaction. Another advantage of DAs is their ability to control conversations about topics and nudge their user in other directions. This puts the system in the position to inform the user about other possible topics. The next step could be a DA that is able to answer for its user based on their usual behavior [48]. On the other hand, there are studies revealing that the users' expectations were not met and mediocre natural language capabilities disappointed and frustrated users [36]. Furthermore, answers from a DA are often rather simple compared to answers from a real person [53].

In the study at hand, we complement this research stream with a perspective on the entire DT process as we seek to understand the potentials and boundaries of DAs as facilitators to support the DT moderator or team. As previously described, DT is a complex process that strongly relies on a skilled facilitator, who knows the methods well, keeps track of the team's dynamic and progress, and guides the team through the task. Although DAs are unlikely to replace expert human facilitators in the near future, they may be able to augment their work, reduce effort, and take over specified parts of the task. This could include for example monitoring balanced activity levels of all team members or the contribution of data-based background information. Therefore, human facilitators could focus on the crucial parts of their work while the team gets more guidance from the DA [67]. Additionally, DAs are neutral instances without an own agenda [67]. Therefore, humans may have fewer obstacles to discuss critical issues as DAs do not convey verbal or nonverbal judgment [49]. Moreover, they are able to generate more knowledge during the design process., especially, if they have access to conversations or internal company information. The DBpedia chatbot for example is used to enhance community interactions by analyzing data of conversations within a community. Thus, the chatbot is able to search conversations to check, if a question has already been answered [6]. Furthermore, the system is able to check external sources to get more relevant information, or information related to the actual search topic [48]. It has to be noted that there is a variety of methods and approaches to design a DA and those techniques are still a matter for debate [1]. Studies explored the extent to which humanlike cues such as the name of the DA or the language style can influence the perception of social presence $[4$, 29]. Because of the hedonic nature, the perceived social presence has been shown to have a positive effect on the perceived enjoyment and ease of use [52, 66]. Rietz, Benke and Maedche [51] investigated the functional and anthropomorphic design features of DAs in collaboration. The result showed that anthropomorphism has a highly significant effect on perceived usefulness. With our work, we complement this empirical and experimental literature and explore general capabilities and characteristics of DAs to provide facilitation assistance throughout entire collaborative creative design processes.

\subsection{Facilitation}

Outcomes of various team collaboration processes have been shown to depend on facilitation, i.e. interventions performed by a facilitator in a collaborative process that guide groups to achieve their common goals $[7,14,40]$. Effective facilitation is affected by the facilitator's skills $[13,17,23]$. Those skills required for complex collaboration processes cover the broad range from keeping the focus of the team on the task toward an optimum outcome, building up a positive dialog environment by observing and intervening in an encouraging way, encouraging participation and controlling the collaborative setting, recognizing individuals as different and approaching them individually [23]. According to the Facilitation Framework [13], facilitative acts can be categorized as either targeted toward the task, the process to accomplish this task or activities that affect the relationship during the process. In a similar way, Dickson et al. [24] classified facilitative acts into task interventions and interactional interventions. Task interventions refer to facilitative acts to direct the group's focus to reach the group goal. Interactional interventions are facilitative acts to improve and stimulate group dynamics and communication by considering its members' socio-emotional state.

Facilitators of collaboration processes such as DT, face the challenge to master both types of facilitative acts, which poses high demands on their training and experience as well as high cognitive load during the collaboration process. A lack of on-demand availability of expert facilitators might thus impede leveraging the potentials of DT in organizations. The "facilitator in a box" idea is one approach to address this bottleneck by transforming facilitative acts to system restrictions with the automated execution of prompts implemented in scripts [17]. However, such systems lack the ability to utilize language skills to construct conversations similar to humans to create a familiar environment for teams when addressing their socio-emotional needs during the collaboration. With the rise of DAs and their increasing functional capabilities, automated facilitation has the potential to become more intuitively integrated into 


\begin{tabular}{|c|c|c|c|}
\hline & & eam & Facilitator \\
\hline $\begin{array}{l}\text { Task and } \\
\text { process } \\
\text { assistance }\end{array}$ & $\begin{array}{l}\text { - Data analysis } \\
\text { - Intelligent research } \\
\text { - Divergence fostering } \\
\text { - Process keeper }\end{array}$ & $\begin{array}{l}\text { - Timekeeper } \\
\text { - Minute taker } \\
\text { - Method explanation }\end{array}$ & $\begin{array}{l}\text { - Workshop feedback analyst } \\
\text { - Participant selection and } \\
\text { invitation } \\
\text { - Method Selection assistance }\end{array}$ \\
\hline $\begin{array}{c}\text { Interaction } \\
\text { assistance }\end{array}$ & $\begin{array}{l}\text { - Team sentiment feedback } \\
\text { - Centrality and speech } \\
\text { share feedback }\end{array}$ & $\begin{array}{l}\text { - Animator / game master } \\
\text { - Workshop feedback }\end{array}$ & $\begin{array}{l}\text { - Facilitation feedback / } \\
\text { - Sparring partner } \\
\text { - Sensing Assistance }\end{array}$ \\
\hline
\end{tabular}

Table 2. Capabilities for digital assistant identified in the interviews

collaborative work practices and should also foster robust and effective DT processes even for less experienced DT coaches and teams. However, due to dynamic technological progress, little is known on how this facilitation support needs to be designed from a practitioner's perspective. Our study contributes by identifying task/process and relationship/interaction facilitation support needs of DT coaches for their teams and themselves. Furthermore, we provide a foundation for further research on the usage of DAs in collaborative, creative design processes.

\section{Research Method}

In order to shed light on facilitation support needs and potentials for DT, we conducted a series of seven expert interviews with DT professionals. We selected a convenience sample of industry experts as well as scholars from the authors' personal networks. Prerequisite for selection was substantial practical DT experience ( $>3$ years) in not only researching or teaching, but moderating DT workshops, as we expect them to know both their own assistance needs as well as those of the broad range of teams they have worked with. We focus on DT as one instance of collaborative, creative processes because it is a well-known and common approach utilized in industry as well as academia. In the semi-structured expert interviews, we asked questions on the interviewees' experience with DT, current needs and problems they face during DT workshops, and questions about potential DA facilitation support. None of the interviewees has been working with DAs in DT yet, which indicates the low prevalence of DAs in this domain and calls for an open, exploratory approach. During the interviews, we followed the imaginative variation interview approach [38] and asked the interviewees to imagine the usage of DAs during their DT workshops. We specifically asked for needed capabilities of and requirements for DAs as well as expected positive or negative outcomes of DA usage during DT workshops.

\begin{tabular}{|l|l|}
\hline No. & Organization and DT Experience \\
\hline I1 & $\begin{array}{l}\text { University teacher and facilitator of student DT } \\
\text { workshops for 5 years }\end{array}$ \\
\hline I2 & $\begin{array}{l}\text { University researcher on DT methods and DT } \\
\text { teacher for 4 years }\end{array}$ \\
\hline I3 & $\begin{array}{l}\text { Innovation project manager and DT facilitator in } \\
\text { large automotive company for 6 years }\end{array}$ \\
\hline I4 & $\begin{array}{l}\text { Strategy consultant and facilitator for corporate } \\
\text { DT workshops for 3 years }\end{array}$ \\
\hline I5 & $\begin{array}{l}\text { University researcher and Consultant on DT } \\
\text { methods for 7 years }\end{array}$ \\
\hline I6 & Founder of DT Agency and Trainer for 6 years \\
\hline I7 & IT consultant with 5 years of DT experience \\
\hline
\end{tabular}

Table 1. Interviewees‘ DT background

The interviewees have an average DT experience of 5.57 years (SD 1.99) and the interviews lasted 53.86 minutes on average (SD 13.67). All interviews were recorded with interviewees consent and paraphrased. Two researchers independently coded the interviews with MAXQDA using an open coding approach [39]. We organized the coding along the questions whom the DA is supporting (the team directly or the human facilitator in the "back-office") and what types of facilitative acts the DA is supporting (task and process or interaction assistance, see section 2.3) and derived desired capabilities inductively. In addition, we identified initial assistance needs that referred to specific DT methods and started to identify future research topics from open issues the interviewees raised. Subsequently, the codes were discussed and refined among the research team and consensus about the coding was reached.

\section{Results}

In the following, we discuss the findings from our interviews (I1 - I7) along the four fields of Table 2 with respect to several capabilities the DA could have to support the DT team and the human facilitator. We use the term capability in the sense of Markus et al. [45] to refer to the ability of the DA to provide a certain functionality, e.g. to provide feedback to the DT team or audio record the content of a team meeting. Table 2 summarizes our results. In our analysis, we focus on the DA as facilitator. The DA could take over further roles, such as peer within the group. As technology is advancing and getting even more intelligent, future DAs can take over 
more roles and responsibilities in collaborative creative design processes, which is out of scope of this study.

\subsection{Team Task and Process Facilitation}

Seven capabilities of DAs were addressed by the interviewees that target the frontline support of the DT team to achieve their goal and execute the DT process. The first three capabilities within this field focus on supporting certain recurring tasks of DT teams, namely analyzing data, acquiring new knowledge, and creating divergent ideas. First, I1, I3, I4, I5 and I7 stated the need for a Data Analysis capability that helps the team to process and make sense of the large amount of data they gather during the DT process, e.g. from interviews or desk research. Potential benefits of such a system are seen in more time efficient and conclusive analysis of large datasets from different perspectives (I3, I7), the higher objectivity compared to human analysis (I1, I3, $\mathrm{I} 4, \mathrm{I5})$, and the revelation of patterns within the data (I3). In a similar way, the DA could not only analyze data provided by the DT team, but make further external data sources accessible to the team with an Intelligent Research capability, which applies AI to present conditioned knowledge upon request (I2, I7) or proactively. The DA could, for example, analyze social media information (I2) conduct market and user research (I3, I5) or general desk research (I4), verify arguments in team discussions with factual data (I3), or access knowledge from prior workshops (I2). Interviewees noted that such knowledge would need to be presented in form that is quickly available, easy to understand and use during the workshop (I7). Moreover, the utilized sources and reasoning process by the DA must be made transparent (I4). The third capability refers to the teams' frequent challenge to get and stay in a creative work mode and think out of the box, which is essential for the DT approach. A Divergence Fostering capability may give creative impulses and encourage participants to express divergent ideas. The DA could induce utterances that participants can build on, e.g. information from social media (I2), example ideas (I2), guiding questions or hints to topics that have not been addressed yet (I5, I6) or experiences from past projects (I5). It could also give procedural guidance on how to work divergently, e.g. by reminding of team rules for creative work (I1) or pointing out biases toward or against certain solution spaces (I3).

The next four capabilities focus on facilitating the DT process as such. On a global level, interviewees requested a Process Keeper capability compassing all facilitative acts that help the team stick to the DT process and execute it in the intended way, including explanations on why a certain step is taken. Such a capability should relieve the facilitator from some monitoring duties and act proactively, e.g. by recognizing and pointing out breaches of rules and deviations from the planned process to the team (I1, I2, I7) as well as by continuously visualizing the DT process and progress of the team (I5, I7). In addition to proactive management of the whole process, Method Explanation was mentioned as a beneficial capability for a reactive DA, as numerous different DT methods exist, and participants are often inexperienced with the overall approach as well as specific methods. The DA should be able to answer participants' questions in relation to specific methods or the current state of the process (I2, I7). This could allow participants to act more independently in situations, in which they need to work alone e.g. during interviews (I5) or in sub teams, where the human facilitator's attention is a bottleneck (I7). A Time Keeper capability was mentioned by I2, I3, and I7 as a simple use case to automate a routine facilitation task and remind the team unobtrusively. 17 noted that sticking to the time plan for the phases is important, as DT is about generating and rejecting ideas quickly instead of focusing on a single idea for too long. Finally, the team process can be supported by a Minute Taker capability. All interviewees stated that the DT sessions they facilitate are predominantly characterized by faceto-face oral discussion as well as paper based hands-on work; digital communication or collaboration tools are hardly used. Thus, teams face the challenge to reconstruct their decision-making processes in retrospective and important information might be lost (I5). A DA could transcribe the session and use natural language processing to make generated knowledge accessible.

\subsection{Team Interaction Facilitation}

The interviews revealed four types of capabilities that refer to a DA helping the team directly to monitor and improve their interpersonal interaction. The most prevalent capability, Team Sentiment Feedback was picked out as a central theme by five out of seven interviewees and relies on tracking the team conversation and sentiment. I1, I2, I3, and I6 see value in a system reminding the team to keep the DT mindset and be open and constructive, if it detects critical or negative utterances or behavior in phases where they are not appreciated. I6 notes that "the assistance system could point out difficult or destructive situations in the discourse, for example: you used the word 'but' three times" (I6, translated). Interviewees can think of different ways how this information is provided, e.g. continuously on an extra screen or as an ad hoc alert. However, they note that the feedback should be unobtrusive to avoid disturbing the creative flow. Furthermore, it should be communicated on a team level to allow for team self-regulation. If individual feedback is 
required, it should be mediated by the human facilitator (I3, I6) to avoid confrontation of individuals, although I1 assumes that critique from a DA might also be better accepted due to its neutrality and objectivity. A second capability that requires natural language understanding of the DA addresses Centrality and Speech Share Feedback. Such a capability allows the DA to inform the team, if one person dominates the conversation or if speech shares are unbalanced between the team members (which might conflict the aspired openness of the DT process and negatively impact participants' satisfaction). The DA could either present speech shares (I3, I6) and leave self-regulation to the team or motivate passive team members directly for turn taking (I2). Such feedback is of importance particularly, if superiors are present or prior team structures manifest (I3, I6, I7). A third capability for team interaction facilitation is enabling the DA to take over the role of an Animator or Game Master, that motivates the team by inducing moodlightening recreational interventions, if participants get exhausted in the course of a long workshop day, such as games, music and jokes (I1, I2, I7). Finally, several facilitators end their DT workshops with collecting feedback from the participants. I6 mentions that this activity results in a need for an automated and interactive Workshop Feedback capability to allow the discussion in the wrap-up phase of the workshop.

\subsection{Facilitator Task and Process Assistance}

Capabilities of the DA to support the facilitator "in the back-office" covered activities from the preparation of DT workshops until their wrap-up and documentation. Initially, a DA could support facilitators in the task of Participant Selection and Invitation. It may reduce the facilitator's effort for workshop preparation and ensure an un-biased composition of teams, e.g. with sufficient diversity (I3) or who do not know each other too well (I7). Facilitators may also benefit from Method Selection Assistance capability. I2, I3, I4, and I7 wish for an intelligent method toolbox or database that helps them to select and configure (e.g. with templates) an effective method based on good practices for the specific task and team setting. This capability should cover the whole DT process and be available upfront during workshop preparation as well as during the workshop, when facilitators flexibly need to adjust their plan in accordance to progress and team dynamics. At the end of the workshop and at certain milestones, facilitators would like to outsource parts of their Process Documentation and Digitalization tasks to a DA, which are seen as high-effort, non-valueadding routine work. The DA could digitize paper-based artefacts (post-it notes, flipcharts, paper prototypes), reduce media discontinuities and make analogous documents available for further digital processing. Automated documentation can also make results and knowledge available across workshops and build up a knowledge base (I2).

\subsection{Facilitator Interaction Assistance}

The interviews revealed two capabilities to help the facilitator execute their interaction-oriented tasks. The first refers to Sensing Assistance for the human facilitator, which continuously monitors certain indicators of team mood (e.g. facial expressions and gestures, language and tonality) and alerts the facilitator in case of negative team dynamics. The need for such support was mentioned in four out of seven interviews. Interviewees stressed in particular the challenge of keeping track of the task, process, and team interaction at the same time and noted that sensing assistance could relieve them from this multitasking challenge to a certain extent. The second capability, Facilitation Feedback by the DA regarding their behavior and facilitation performance to learn and improve, was requested by two interviewees. They appreciate workshops with co-facilitators and see a potential benefit in an objective DA to act as a sparring partner where no other human colleague is available.

\subsection{Method Specific Assistance}

Surprisingly, most of the facilitation needs raised by the interviewees did not target a specific method or technique, but experts rather asked for continuous support throughout the complete DT process. However, for some methods, a DA could provide specialized functionality. The interviews revealed that DAs can be particularly useful for conducting focus groups (Focus Group Facilitation). Focus groups might be conducted online with partly automated facilitation of a DA, which could enable the involvement of an increased number of participants under limited human facilitator availability. Furthermore, a speech-to-text capability of a DA could be used as an extension of human participants and support other participants in writing ideas down. A further capability of the DA could be supporting interviews by automatically transcribing and analyzing the interviews (Interview Transcript Analyst). The DA should be able to detect patterns and reveal hidden insights. Here, the DA might cluster topics and structure information within texts (Topic Clustering and Information Structuring). Lastly the interviews revealed that a DA might be particularly helpful for brainstorming sessions. During brainstorming sessions, humans tend to throw around every thought, which comes to their mind, which may lead to a chaotic situation. Thus, after such a session, the DA could prepare and categorize the ideas and deliver a compact summary, enabling 
objective decision making. Furthermore, it could foster different directions, by providing impulses and bring in new ideas (Brainstorming Seeds). Participants might build upon these impulses and develop the idea further.

\section{Research Agenda}

Based on our analysis of the interviews, we identified six avenues for future research on the design of DA for collaborative, creative design processes such as DT. In the following, we outline these topics with more detail. The first stream of research addresses the capabilities of the DA. Interviewees had some concerns about the capabilities and the resulting performance of the system, especially when "the system is not sophisticated, and the answers are therefore not helpful, but frustrating" (I7, translated). Thus, an important avenue for future research is to further investigate users' needs and requirements for such DAs with respect to their functionality in light of the fast-changing technology landscape. Based on these findings, the design of DAs to fulfil these requirements needs to be investigated and prescriptive design knowledge contributed. The second topic addresses the opportunity to combine human and non-human facilitators to enhance the capabilities of the DA by human in the loop learning. Many interviewees (I2, I3, I6, and I7) suggested the functionality to rate the actions by the DA and provide feedback to enable the system to learn. Future research could explore how the feedback could be collected efficiently and with as little effort as possible. Moreover, research should explore what kind of feedback is required (simple ratings or more complex qualitative feedback). In general, a division of labor among human and DA facilitator that leverages their unique strengths and allows for seamless handovers, remains an important research field to investigate. The third avenue for future research addresses the three interrelated topics security, privacy, and ethics. Especially the recording and processing of personrelated information and the effects on the acceptance of the DA was mentioned as an important challenge by two interviewees (I2 and I6). Moreover, the utilization of DAs was questioned in general, for example by $\mathrm{I} 3$ who stated that to "blindly rely on the system is an ethical question" ( 13 , translated). This research stream might be of special importance for creative work such as DT, where the interviewees appeared to identify strongly with their profession and where DAs might enter the very heart of their job. The fourth avenue for future research addresses the DAs' impact on team collaboration, which was an important aspect for all interviewees. Thereby, future research could, for example, investigate how the DA affects team dynamics (I1, I5), if team members feel inhibited or encouraged when a
DA with a conversational interface is present (I2, I3), or if a DA positively or negatively impacts the team's creativity (I4, I3, I5, I6). The fifth avenue for future research addresses the role, agency, and authority of the DA and was discussed very intensively by all interviewees. Future research should, for example, explore, if the DA should be an active or passive team member (I1, I5, I6) or should explicitly not serve as a team member (I4). Moreover, future research could investigate, how human team members perceive the authority of the DA, how the presence of a DA impacts the human facilitator's authority and analyze the resulting impact on the team's behavior (I1, I2, I7). Similarly, research could explore how the neutrality and objectivity of a DA affects the team, which consists of humans having subjective opinions and behavior (I3). The sixth avenue for future research addresses the appearance of the DA. Research should explore, to which degree the DA should be designed to be humanlike (I2, I7). Moreover, research should investigate the questions, if a (virtual and / or physical) embodiment of the DA is required or has a negative impact (I2, I5). Many interviewees (I2, I3, I4, I5) stressed the importance of having a voice-based interface for interaction during the workshop and future research could investigate how to structure the dialog between team and DA using voice-based interfaces. However, interviews also suggested that different modes of interaction might be necessary in different phases and tasks, e.g. the facilitator needs to get discreet alerts during the workshop.

\section{Conclusion, Limitations and Outlook}

Design spaces for useful and performant DAs for complex and collaborative human tasks are opening up due to advances in AI technologies. In the study at hand, we gathered and structured an initial understanding of the potential design space for DA facilitation support for collaborative, creative design processes such as DT. The interviews revealed that DT coaches see great potential for augmenting and assisting their own and their teams' efforts with facilitation support by a DA, both for process/task-oriented interventions as well as for interaction facilitation. However, it also becomes clear that DA capabilities and how they are expressed in collaboration need to be carefully crafted to enhance rather than inhibit collaboration and creativity. From the expressed needs by seven experienced DT coaches, we identified 16 distinct capabilities of DAs that can guide design research and practitioners' endeavors toward helpful automated DT facilitation support. We also contribute initial hints on method-specific support as well as a first research agenda outlining six avenues for further research. Follow-up research can build on this work by investigating, how the presence of DA 
facilitators, different characteristics and interventions of such DAs may impact relevant performance metrics of creative teams, such as productivity or creativity of solutions, which exceeds the scope of this paper. Furthermore, our study results provide indication that individual or team level process variables, e.g. motivation, trust, cognitive load, or psychological safety might depend on DA involvement. Future research should seek to understand these relationships to provide descriptive and prescriptive knowledge for human-DAteamwork.

The presented findings need to be considered with adequate caution due to the early stage of the research field and the inherent limitations of the exploratory study. First, DT facilitation needs, and digitalization potentials might vary with the available infrastructure, the type of artefacts to be designed, and the nature of the teams involved. While all interviewees are experienced experts in DT and contributed rich insights, the sample size should be extended towards further DT coaches from different industries and within different settings. Second, the picture needs to be complemented with the first-hand perspective from DT participants, who are only represented indirectly via their DT coaches' voices in the current data. Third, due to the innovative nature of the topic, all expressed needs, expectations, and attitudes toward DA facilitation support were based on imagination, as no DAs are at use yet in the DT coaches' work. Their ideas might thus be biased by personal experiences with DAs from other contexts or the lack thereof. Thus, an important next step to validate the findings from this study is to instantiate the most promising potential capabilities and investigate their functioning and impact in laboratory and real-world DT processes.

\section{References}

[1] Abdul-Kader, S.A., and J. Woods, "Review on Chatbot Design Techniques in Speech Conversation Systems", Iarjset 5(9), 2018, pp. 37-46.

[2] Abu Shawar, B., and E. Atwell, "Chatbots: are they really useful?", LDV-Forum: Zeitschrift für Computerlinguistik und Sprachtechnologie 22(1), 2007, pp. 29-49.

[3] Ambrose, G., and P. Harris, "Design Th! Nking Th! Nking", 2010, pp. 1-199.

[4] Araujo, T., "Living up to the chatbot hype: The influence of anthropomorphic design cues and communicative agency framing on conversational agent and company perceptions", Computers in Human Behavior 85, 2018, pp. $183-189$.

[5] Armstrong, C.E., "Teaching Innovation Through Empathy", Management Teaching Review 1(3), 2016.

[6] Athreya, R.G., A.-C. Ngonga Ngomo, and R. Usbeck, "Enhancing Community Interactions with Data-Driven Chatbots--The DBpedia Chatbot", (2018).
[7] Bittner, E., and J. Leimeister, "Creating shared understanding in heterogeneous work groups: Why it matters and how to achieve it", Journal of Management Information Systems 31(1), 2014, pp. 111-144.

[8] Bittner, E., S. Oeste-Reiß, and J.M. Leimeister, "Where is the Bot in our Team? Toward a Taxonomy of Design Option Combinations for Conversational Agents in Collaborative Work", Proceedings of the 52nd Hawaii International Conference on System Sciences(January), 2019.

[9] Bittner, E., and O. Shoury, "Designing Automated Facilitation for Design Thinking: A Chatbot for Supporting Teams in the Empathy Map Method", Proceedings of the 52nd Hawaii International Conference on System Sciences, 2019, pp. 227-236.

[10] Bittner, E.A.C., G.C. Küstermann, and C. Tratzky, "The facilitator is a bot: Towards a conversational agent for facilitating idea elaboration on idea platforms", 27th European Conference on Information Systems Information Systems for a Sharing Society, ECIS 2019, (2020).

[11] Bjögvinsson, E., P. Ehn, and P.A. Hillgren, "Design things and design thinking: Contemporary participatory design challenges", Design Issues 28(3), 2012.

[12] Blackburn, T., P. Swatman, and R. Vernik, "Cognitive dust: Linking CSCW theories to creative design processes", Proceedings - 2006 10th International Conference on Computer Supported Cooperative Work in Design, CSCWD 2006, (2006).

[13] Bostrom, R.P., R. Anson, and V.K. Clawson, "Facilitation and group support systems", In L. Jessup and J. Valchich, eds., Group Facilitation and Group Support Systems. Macmillan, New York, 1993, 146-168.

[14] Bowers, C.A., J.A. Pharmer, and E. Salas, "When member homogeneity is needed in work teams: A metaanalysis", Small Group Research 31(3), 2000, pp. 305327.

[15] Brachten, F., M. Mirbabaie, S. Stieglitz, O. Berger, S. Bludau, and K. Schrickel, "Threat or opportunity? Examining social bots in social media crisis communication", ACIS 2018 - 29th Australasian Conference on Information Systems, (2018), 1-8.

[16] Brandtzaeg, P.B., and A. Følstad, "Why people use chatbots", Lecture Notes in Computer Science (including subseries Lecture Notes in Artificial Intelligence and Lecture Notes in Bioinformatics) 10673 LNCS, 2017, pp. 377-392.

[17] Briggs, R.O., G.L. Kolfschoten, G.J. De Vreede, S. Lukosch, and C.C. Albrecht, "Facilitator-in-a-box: Process support applications to help practitioners realize the potential of collaboration technology", Journal of Management Information Systems 29(4), 2013, pp. 159194.

[18] Buhl, A., M. Schmidt-Keilich, V. Muster, et al., "Design thinking for sustainability: Why and how design thinking can foster sustainability-oriented innovation development", Journal of Cleaner Production 231, 2019, pp. 1248-1257.

[19] Burton, N., and J. Gaskin, "“Thank you, siri’: Politeness and intelligent digital assistants", 25th Americas Conference on Information Systems, AMCIS 2019, 
(2019).

[20] Canedo, E.D., and F.V. Almeida, "A Study on the Design Thinking Approach in Universities", Proceedings of the $X V$ Brazilian Symposium on Information Systems SBSI'19, ACM Press (2019), 1-8.

[21] Cautela, C., M. Mortati, C. Dell'Era, and L. Gastaldi, "The impact of artificial intelligence on design thinking practice: Insights from the ecosystem of startups", Strategic Design Research Journal 12(1), 2019, pp. 114134.

[22] Chon, H., and J. Sim, "From design thinking to design knowing: An educational perspective", Art, Design and Communication in Higher Education 18(2), 2019.

[23] Clawson, V.K., and R.P. Bostrom, "Research-driven facilitation training for computer-supported environments", Group Decision and Negotiation 5(1), 1996, pp. 7-29.

[24] Dickson, G.W., J.E. Lee-Partridge, M. Limayem, and G.L. Desanctis, "Facilitating computer-supported meetings: A cumulative analysis in a multiple-criteria task environment", Group Decision and Negotiation 5(1), 1996, pp. 51-72.

[25] Diederich, S., A.B. Brendel, and L.M. Kolbe, "On Conversational Agents in Information Systems Research: Analyzing the Past to Guide Future Work", Proceedings of the International Conference on Wirtschaftsinformatik, 2019, pp. 1550-1564.

[26] Dobrigkeit, F., and D. De Paula, "Design thinking in practice: Understanding manifestations of design thinking in software engineering", ESEC/FSE 2019 Proceedings of the 2019 27th ACM Joint Meeting European Software Engineering Conference and Symposium on the Foundations of Software Engineering, 2019, pp. 1059-1069.

[27] Ewin, N., J. Luck, R. Chugh, and J. Jarvis, "Rethinking Project Management Education: A Humanistic Approach based on Design Thinking", Procedia Computer Science, (2017).

[28] Fabri, M., "Thinking with a New Purpose: Lessons Learned from Teaching Design Thinking Skills to Creative Technology Students", Design, User Experience, and Usability: Design Discourse, Springer International Publishing (2015), 32-43.

[29] Feine, J., U. Gnewuch, S. Morana, and A. Maedche, "A Taxonomy of Social Cues for Conversational Agents", International Journal of Human-Computer Studies 132, 2019, pp. 138-161.

[30] Frommert, C., A. Häfner, J. Friedrich, and C. Zinke, "Using Chatbots to Assist Communication in Collaborative Networks", IFIP Advances in Information and Communication Technology, (2018).

[31] Geissdoerfer, M., N.M.P. Bocken, and E.J. Hultink, "Design thinking to enhance the sustainable business modelling process - A workshop based on a value mapping process", Journal of Cleaner Production 135, 2016.

[32] Gnewuch, U., S. Morana, and A. Maedche, "Towards Designing Cooperative and Social Conversational Agents for Customer Service", Proceedings of the International Conference on Information Systems (ICIS), (2017).
[33] Hajare, A., P. Bhosale, R. Nanaware, and G. Hiremath, "Chatbot for Education System", International Journal of Advance Research, Ideas and Innovations in Technology ISSN: 4(3), 2018, pp. 37-43.

[34] Henriksen, D., C. Richardson, and R. Mehta, "Design thinking: A creative approach to educational problems of practice", Thinking Skills and Creativity 26, 2017.

[35] Ih-cheng, L.A.I., "Overlap Real And Virtual Design Worlds", 2001, pp. 114-117.

[36] Jain, M., P. Kumar, R. Kota, and S.N. Patel, "Evaluating and informing the design of chatbots", DIS 2018 Proceedings of the 2018 Designing Interactive Systems Conference, 2018, pp. 895-906.

[37] Kim, S., J. Eun, C. Oh, B. Suh, and J. Lee, "Bot in the Bunch: Facilitating Group Chat Discussion by Improving Efficiency and Participation with a Chatbot", (February), 2020, pp. 1-13.

[38] King, N., C. Horrocks, and J. Books, Interviews in Qualitative Research, SAGE Publications, Inc, 2019.

[39] Kuckartz, U., "Qualitative Text Analysis: A Systematic Approach", In G. Kaiser and N. Presmeg, eds., Compendium for Early Career Researchers in Mathematics Education. Springer, Cham, 2019, 181197.

[40] Langan-Fox, J., J. Anglim, and J.R. Wilson, "Mental Models, Team Mental Models, and Performance: Process, Development, and Future Directions", Human Factors and Ergonomics in Manufacturing 14(4), 2004, pp. 331-352.

[41] Laumer, S., F. Gubler, A. Racheva, and C. Maier, "Use cases for conversational agents: An Interview-based Study", 25th Americas Conference on Information Systems, AMCIS 2019, (2019).

[42] Liedtka, J., "Perspective: Linking Design Thinking with Innovation Outcomes through Cognitive Bias Reduction", Journal of Product Innovation Management 32(6), 2015, pp. 925-938.

[43] Lugmayr, A., B. Stockleben, Y. Zou, S. Anzenhofer, and M. Jalonen, Applying "design Thinking" in the context of media management education, 2014.

[44] Maedche, A., C. Legner, A. Benlian, et al., "AI-Based Digital Assistants", Business \& Information Systems Engineering 61(4), 2019, pp. 535-544.

[45] Markus, M.L., A. Majchrzak, and L. Gasser, "Management Information Systems Research Center, University of Minnesota", MIS Quarter 26(3), 2002, pp. 179-212.

[46] Micheli, P., S.J.S. Wilner, S.H. Bhatti, M. Mura, and M.B. Beverland, "Doing Design Thinking: Conceptual Review, Synthesis, and Research Agenda", Journal of Product Innovation Management 36(2), 2019, pp. 124148.

[47] Omale, G., "Gartner Predicts 25 Percent of Digital Workers Will Use Virtual Employee Assistants Daily by 2021",

[48] Paikari, E., and A. Van Der Hoek, "A framework for understanding chatbots and their future", Proceedings International Conference on Software Engineering, 2018, pp. 13-16.

[49] Pickard, M.D., C.A. Roster, and Y. Chen, "Revealing sensitive information in personal interviews: Is self- 
disclosure easier with humans or avatars and under what conditions?", Computers in Human Behavior 65, 2016.

[50] Rauth, I., L. Carlgren, and M. Elmquist, "Making It Happen: Legitimizing Design Thinking in Large Organizations", Design Management Journal 9(1), 2014, pp. 47-60.

[51] Rietz, T., I. Benke, and A. Maedche, "The Impact of Anthropomorphic and Functional Chatbot Design Features in Enterprise Collaboration Systems on User Acceptance", 14th International Conference on Wirtschaftsinformatik(February), 2019, pp. 1642-1656.

[52] Rodrigues, L.F., A. Oliveira, and C.J. Costa, "Does easeof-use contributes to the perception of enjoyment? A case of gamification in e-banking", Computers in Human Behavior 61, 2016, pp. 114-126.

[53] Rosé, C.P., C. Torrey, and V. Aleven, "Guided Exploratory Learning versus Directed Learning in a Simulation Environment for Thermodynamics: A Pilot Study", ITS 2004 Workshop Proceedings on Dialogbased Intelligent Tutoring Systems, (2004), 43-48.

[54] Saenz, J., W. Burgess, E. Gustitis, A. Mena, and F. Sasangohar, "The usability analysis of chatbot technologies for internal personnel communications", 67th Annual Conference and Expo of the Institute of Industrial Engineers 2017, 2017, pp. 1357-1362.

[55] Sándorová, Z., T. Repáňová, Z. Palenčíková, and N. Beták, "Design thinking - A revolutionary new approach in tourism education?", Journal of Hospitality, Leisure, Sport and Tourism Education 26(February 2019), 2020, pp. 100238.

[56] Schleinkofer, U., T. Herrmann, I. Maier, T. Bauernhansl, D. Roth, and D. Spath, "Development and evaluation of a design thinking process adapted to frugal production systems for emerging markets", Procedia Manufacturing 39, 2019, pp. 609-617.

[57] Seeber, I., E. Bittner, R.O. Briggs, et al., "Machines as teammates: A research agenda on AI in team collaboration", Information and Management 57(2), 2020 , pp. 103174.

[58] Seeber, I., L. Waizenegger, S. Seidel, S. Morana, I. Benbasat, and P.B. Lowry, "Collaborating with Technology-based Autonomous Agents: Issues and research opportunities", Internet Research 30(1), 2020, pp. 1-18.

[59] Sonnenburg, S., "Creativity in communication: A theoretical framework for collaborative product creation”, Creativity and Innovation Management 13(4), 2004, pp. 254-262.

[60] Stockleben, B., M. Thayne, S. Jäminki, et al., "Towards a framework for creative online collaboration: A research on challenges and context", Education and Information Technologies 22(2), 2017.

[61] Stoeckli, E., C. Dremel, F. Uebernickel, and W. Brenner, "How affordances of chatbots cross the chasm between social and traditional enterprise systems", Electronic Markets, 2019.

[62] Strohmann, T., D. Siemon, and S. Robra-Bissantz, "brAInstorm: Intelligent Assistance in Group Idea Generation", 10243, 2017, pp. 457-461.

[63] Tham, J., "The Scalability of Design Thinking in Technical and Professional Communication Programs: Two Course Deployment Examples", Proceedings for 2018 CPTSC Annual Conference, (2018), 68-69.

[64] Trippas, J.R., D. Spina, F. Scholer, et al., "Learning About Work Tasks to Inform Intelligent Assistant Design", Proceedings of the 2019 Conference on Human Information Interaction and Retrieval, ACM (2019), 514.

[65] Tsai, P., "Data snapshot: AI Chatbots and Intelligent Assistants in the Workplace - Spiceworks", 2018.

[66] Turel, O., A. Serenko, and N. Bontis, "User acceptance of hedonic information technologies: A perceived value perspective", Association for Information Systems - 13th Americas Conference on Information Systems, AMCIS 2007: Reaching New Heights 3, 2007, pp. 1705-1715.

[67] Wang, H.C., C.P. Rosé, Y. Cui, C.Y. Chang, C.C. Huang, and T.Y. Li, "Thinking hard together: The long and short of collaborative idea generation in scientific inquiry", Computer-Supported Collaborative Learning Conference, CSCL 8(PART 2), 2007, pp. 753-762.

[68] Winkler, R., M.L. Neuweiler, E. Bittner, and M. Söllner, "Hey alexa, please help us solve this problem! How interactions with smart personal assistants improve group performance", 40th International Conference on Information Systems, ICIS 2019, (2020), 1-17.

[69] Wolniak, R., "The Design Thinking method and its stages", Systemy Wspomagania w Inżynierii Produkcji 6(6), 2017, pp. 247-255.

[70] Xu, A., Z. Liu, Y. Guo, V. Sinha, and R. Akkiraju, "A new chatbot for customer service on social media", Conference on Human Factors in Computing Systems Proceedings 2017-May(May), 2017, pp. 3506-3510.

[71] Yin, Y. chao, L. fu Sun, and C. Guo, "A policy of conflict negotiation based on fuzzy matter element particle swarm optimization in distributed collaborative creative design”, CAD Computer Aided Design 40(10-11), 2008.

[72] Zhu, L., B.R. Barricelli, and C. Iacob, "A meta-design model for creative distributed collaborative design", International Journal of Distributed Systems and Technologies 2(4), 2011. 